\title{
Information Visualization and the Challenge of Universal Usability
}

Catherine Plaisant, University of Maryland

plaisant@cs.umd.edu

FINAL VERSION WILL APPEAR as Chapter 3 in

J. Dykes, A.M. MacEachren, M.-J. Kraak (Editors), Exploring Geovisualization, Elsevier, 2005

\begin{abstract}
Information Visualization aims to provide compact graphical presentations and user interfaces for interactively manipulating large numbers of items. We present a simple "data by tasks taxonomy" then discuss the challenges of providing universal usability, with example applications using geo-referenced data. Information Visualization has been shown to be a powerful visual thinking or decision tool but it is becoming important for services to reach and empower every citizen. Technological advances are needed to deal with user diversity (age, language, disabilities, etc.) but also with the variety of technology used (screen size, network speed, etc.) and the gaps in user's knowledge (general knowledge, knowledge of the application domain, of the interface syntax or semantic). We present examples that illustrate how those challenges can be addressed.
\end{abstract}

\subsection{Introduction}

Designers are discovering how to use rapid and high-resolution color displays to present and manipulate large amounts of information in compact and user-controlled ways. Information Visualization can be defined as the use of computer-supported interactive visual representation of abstract data to amplify cognition (Card et al., 1999). The abstract characteristic of the data is what distinguishes Information Visualization from scientific visualization. Information Visualization is more likely to be used to display database content (for example, recorded stock values, health statistics) than.output of models or simulations, but this distinction is not always important. The display of geo-referenced data is often a hybrid visualization that combines abstract and concrete data. In fact several of the most famous examples of Information Visualization include maps, from the 1861 representation of the ill-fated Napoleon's Russian campaign by Minard (see Tufte (1983) and Kraak (undated)) to the interactive HomeFinder application shown in Figure 3.1 that introduced the concept of dynamic queries (Ahlberg et al., 1992). Information Visualization aims to provide compact graphical presentations and user interfaces for interactively manipulating large numbers of items $(102-106)$, possibly extracted from far larger datasets (Card et al., 1999; Spence, 2001; Ware, 2000; Chen, 2002; Bederson and Shneiderman, 2003). Also sometimes called visual datamining, it uses the enormous visual bandwidth and the remarkable human visual system to enable users to make discoveries, take decisions, or propose explanations about patterns, groups of items, or individual items. Perceptual psychologists, statisticians, and graphic designers (Tufte, 1983) offer valuable advice about presenting static information, but advances in processor speed, graphic devices and dynamic displays takes user-interface designers well beyond current wisdom.

This chapter presents a simple "data by tasks taxonomy" then discusses the challenges of providing "universal usability”, with example applications using georeferenced data. 


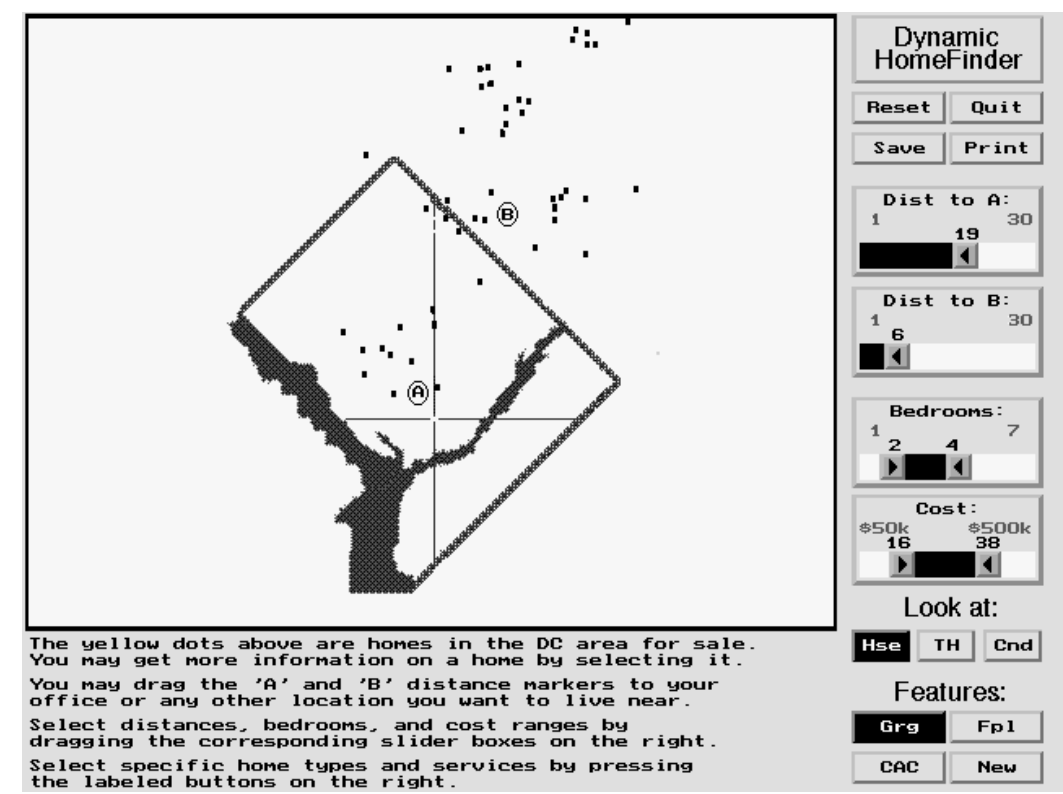

Figure 3.1. The HomeFinder. Houses for sale appear as yellow dots on a stylized map of Washington DC. As users adjust sliders and click on buttons to set their search criteria immediate feedback (within $10 \mathrm{~ms}$ ) is provided on the map showing the houses that match the query, and allowing users to pose hundreds of queries in a few seconds and rapidly explore the dataset.

\subsection{Data by Task Taxonomy}

This data by tasks taxonomy includes seven basic data types and seven basic tasks (Shneiderman, 1998). The data being explored are usually multi-dimensional but most designs highlight 1 , 2 or 3D that are used to define the general visual structure of the visualization. The taxonomy is organized based on those selected dimensions. This simplification is useful to describe the visualizations that have been developed and to characterize the types of problems that users encounter when using those visualizations. Examples were chosen to complement those provided by Keim et al., this volume (Chapter 2) in which a more comprehensive taxonomy for visualization of high dimensional data is the primary focus.

\subsubsection{Data types}

1- 2- or 3D data: Linear data types (1D) include lists, documents, program source code, and the like that are organized sequentially. Interface-design issues include what overview, scrolling, or selection methods can be used. User tasks might be to find the number of items or to see items that exhibit certain attributes (Eick et al., 1992). Planar data (2D) can be represented by geographic maps, floor plans, and newspaper layouts. User tasks are to find adjacent items, containing items and paths between items, and to perform the seven basic tasks (see below). Real-world 3D objects such as the human body or buildings have volumetric elements and connections with other elements. Users' tasks deal with adjacency plus above-below and inside-outside relationships. In 3D applications, users must understand and control their position and orientation when viewing the objects, and must be able to compensate for the serious problems of occlusion. While many examples of successful 3D computer graphics and scientific visualizations exist (Nielson et al., 1997) there are still very few Information Visualization examples in three (abstract) dimensions. Designers have used 3D representations to present attractive overviews of data that was not intrinsically 3D, such as sets of documents, as shown in Figure 3.2 (Wise et al., 1995). But controlled experiments trying to measure the benefits of 3D in such conditions have had mixed results (Cockburn and McKenzie, 2002). These mixed results complement similar findings from earlier cartographic experiments and related efforts to assess use of the third dimension to depict non-spatial attributes on maps (Kraak, 1989; Dorling, 1992)

Temporal data: Time series are very common and merit a data type that is separate from 1D data. The distinctions are that items have a start and finish time, and may overlap. Timelines have been widely used, 
from the line plots used by Minard see Tufte, 1983) to summaries of heterogeneous data such as LifeLines, as shown in Figure 3.3 (Plaisant et al., 1996). Frequent tasks include finding all events before, after, or during some time period or moment, and in some cases comparing periodical phenomena (Carlis and Konstan, 1998). Space-time data have also been a focus of attention in geovisualization for more than a decade (Szego, 1987; DiBiase et al., 1992; Kraak and MacEachren, 1994; Kwan, 2000) and some recent advances are discussed by Andrienko et al., this volume (Chapter 10).

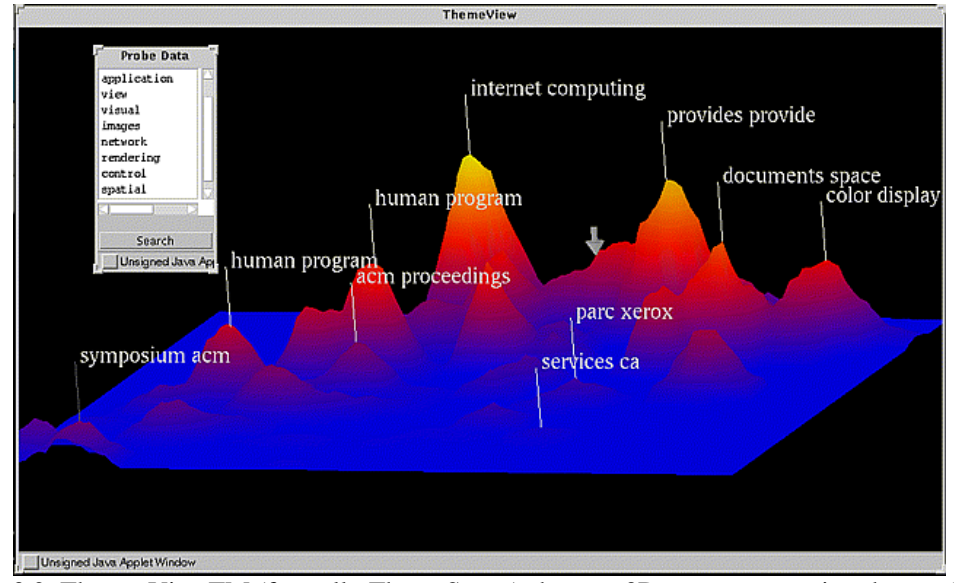

Figure 3.2. ThemesViewTM (formally ThemeScape) shows a 3D map representing the results of a search in a large corpus of documents. Proximity indicates similarity of the topics, whilst height reflects the number of documents and frequency of terms. Commercial applications also exist (OmniViz Inc, 2003) Reproduced with permission of Pacific Northwest National Laboratory.

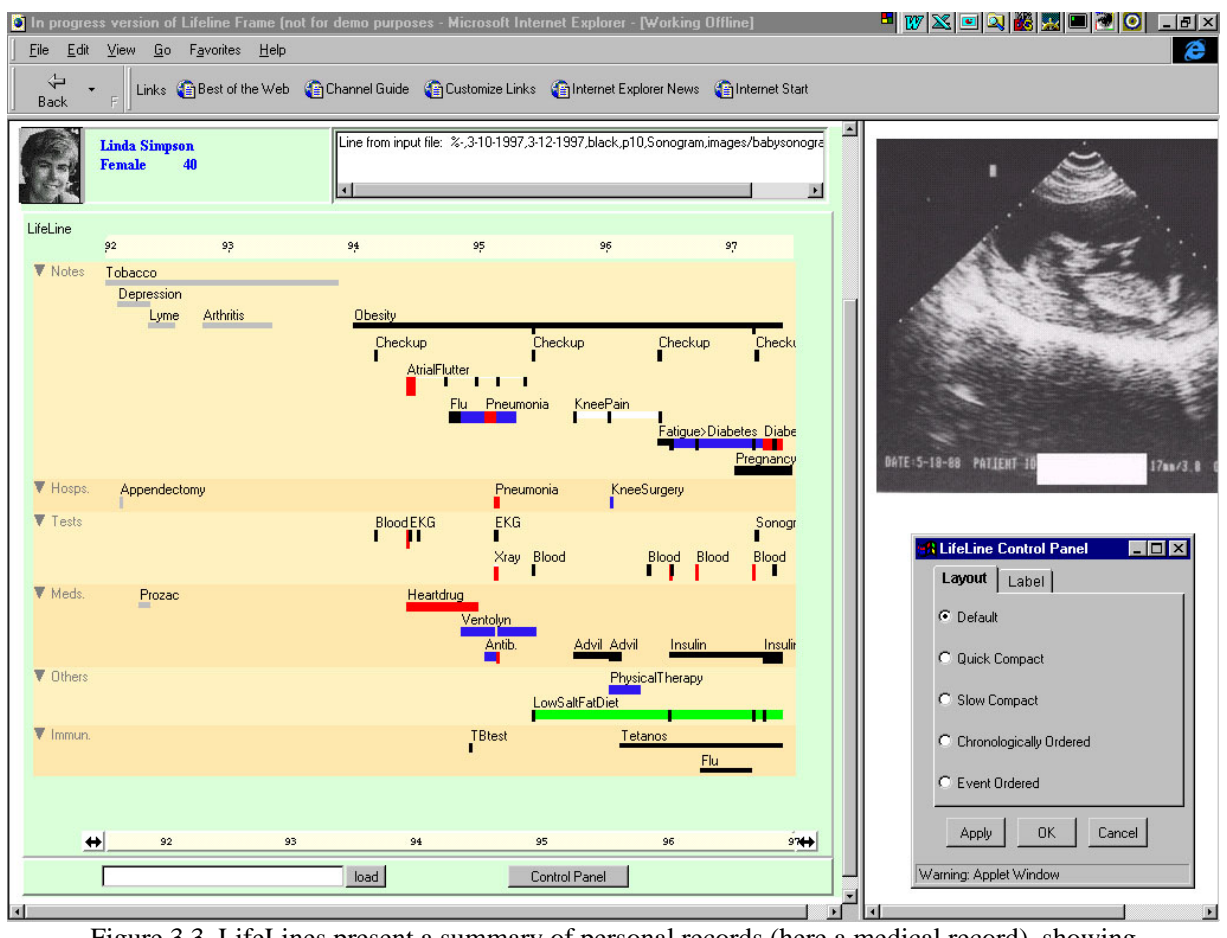

Figure 3.3. LifeLines present a summary of personal records (here a medical record), showing several facets of the records and using line thickness and color to map data attributes on the display. 
Multi-dimensional data: Most relational- and statistical-database contents are conveniently manipulated as multi-dimensional data, in which items with $\mathrm{n}$ attributes become points in an $\mathrm{n}$-dimensional space. The interface representation can be dynamic 2D scattergrams (possibly a map, as is the case in Figure 3.1) with each additional dimension controlled by a slider or button using dynamic queries (Ahlberg et al., 1992). A 3D scattergram is possible, but disorientation and occlusion are severe problems. Parallel coordinates plots (Inselberg, 1985) are one of the few truly multi-dimensional techniques and have been shown to be a powerful analysis tool. Familiarity, training and practice in using the technique will help a user become a "multi-dimensional detective" (Inselberg, 1997). Less powerful but more accessible to novice users is the Table Lens (Rao and Card, 1994; Inxight Software Inc., 2002), which uses a spreadsheet metaphor. Other examples include VisDB for multi-dimensional database visualization (Keim and Kriegel, 1994), interactive mosaic displays (Friendly, 1994; Theus, 2002a,b), the Attribute Explorer (Tweedie et al., 1996) and the scatter plot or prosection matrices of Becker and Cleveland (1987). Interactive geovisualization software also utilizes multidimensional visualization techniques (Andrienko and Andrienko, 1999a-f; Gahegan et al., 2002a,b; MacEachren et al., 2003a,b) as emphasized in section B of this volume (see Andrienko et al., this volume (Chapter 5) and subsequent chapters).

Hierarchical data: Hierarchies or tree structures are collections of items, in which each item (except the root) has a link to one parent item. Examples includetaxonomies, file structures, organization charts and disease classifications. Items and the links between parent and child can have multiple attributes. Tasks can be topological (for example, a query asking which branch of the company has more employees?) or attribute based (such as an attempt to find the largest old files on a hard disk). Interface representations of trees can use the outline style of indented labels used in tables of contents, node-and-link diagrams. Examples include the Hyperbolic tree (Lamping et al., 1995) commercialized by Inxight Software Inc (2002) or SpaceTree (Plaisant et al., 2002; Grosjean et al., 2002). A third possibility is to use a space filling representation such as Treemap (Johnson and Shneiderman, 1991; Bederson et al., 2002) as shown in Figure 3.4. A number of commercial applications of the Treemap technique are available including Shneiderman (1998), the Map of the Market (SmartMoney.com, 2003) or e-business (The Hive Group Inc., 2003).

Network data: When relationships among items cannot be captured conveniently with a regular tree structure, items are linked to an arbitrary number of other items in a network. In addition to performing the basic tasks applied to items and links, network users often want to know about shortest or least costly paths connecting two items or traversing the entire network. Common representations include node-andlink diagrams (but layout algorithms are often so complex that user interaction remains limited for large networks), and square matrices of items with the value of a link attribute in the row and column representing a link. Network visualization is an old but still imperfect art because of the complexity of relationships and user tasks, for example, (see Rodgers, this volume (Chapter 7)). It is used in a number of useful geographic applications and is being incorporated into software for geovisualization, for examples, (see Mountain, this volume (Chapter 9)) and Fairbairn, this volume (Chapter 26).



Figure 3.4. An example of Treemap is the Map of the Market (SmartMoney.com, 2003). Rectangles

each represent a stock and are organized by industry groups. The rectangle size is proportional to the market capitalization and the color indicates the percentage gain or loss for the given time period. Reproduced with permission of SmartMoney.com. 


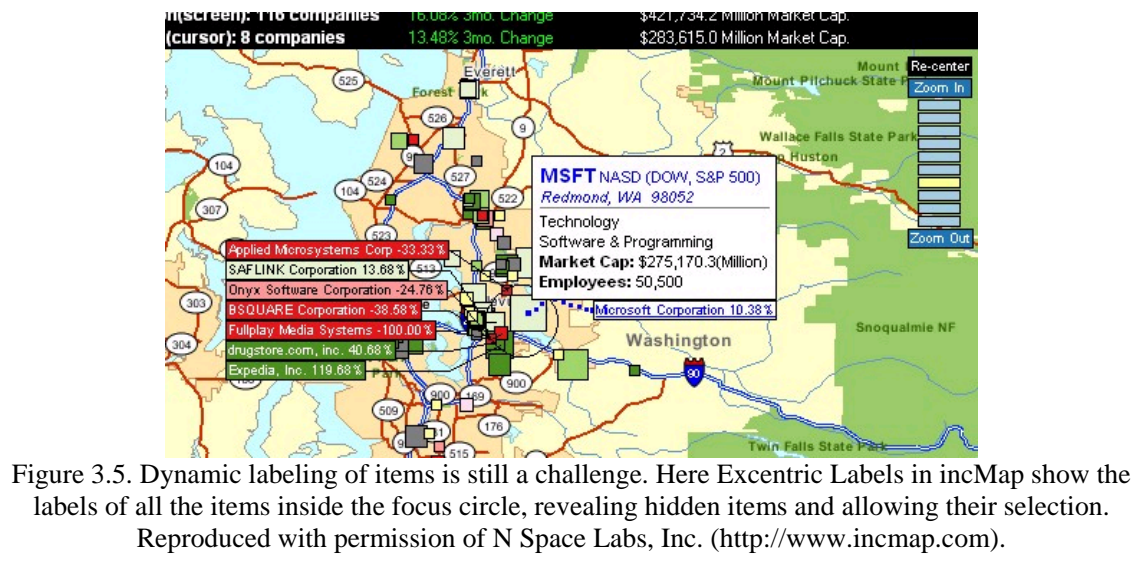

\subsubsection{High-level task types}

Having considered the range of data types available along with some methods that have been developed for graphically representing them, we can consider a number of highlevel tasks that apply to all data types

Overview task: Gaining an overview of the data might include gauging the number of items and the range and distribution of the attribute values, or estimating how much things have changed since last time the user reviewed the data. Overview strategies include zoomed-out views adjoining the detail views, for example, (see Ware and Plumlee, this volume (Chapter 29)). A movable field-of-view box can be used to control the contents of the detail view. Intermediate views allow larger zoom factors. Another popular approach is the fisheye strategy originally described by Furnas (1986). It provides overview and details in a single combined view by using distortion based on a degree of interest function. It is effective when zoom factors are small and deformation is acceptable to users (for example, when orientation and distance measurement are not important).

Zoom task: Users need to control the zoom focus and the zoom factor. Smooth zooming helps users to preserve their sense of position and context. Manual zooming is powerful but users tend to get lost so application-controlled zooming to preset levels is more likely to be usable, for example, see Ware and Plumlee, this volume (Chapter 29). Pad++, now called Piccolo is a popular zooming user interface toolkit that uses semantic zooming (Bederson, 1994; Bederson et al., 2000). Semantic zooming (Perlin and Fox, 1993) is commonly used with maps, where the same area can be displayed with different features and amount of details at different zoom ratios. There are key parallels with real time cartographic generalization here (Weibel and Jones, 1998). Constant density zooming (Woodruff et al., 1998) is an example of technique to maximize the number and readability of items on the display. Wood, this volume (Chapter 15) uses mipmapping to display surface characteristics according to the scale at which any part of a surface is viewed in a real-time 3D application and Doellner, this volume (Chapter 16) identifies some associated issues in computer graphics.

Filter task: Dynamic queries allow users to quickly focus on their interests by eliminating unwanted items. Other techniques include sorting, grouping or highlighting followed by hiding, or locating items similar to an item of interest. Theus, this volume (Chapter 6) provides some examples of "selection" from the perspective of statistical graphics.

Details-on-demand task: Once a collection has been trimmed, users need to review the details of single items or groups. The usual approach is to simply click on an item and review details in a separate or popup window. Dynamic labeling remains an important challenge. Excentric labeling (Fekete and Plaisant, 1999) is an approach illustrated in Figure 3.5 through the Geographic Map of the Market in which geovisualization techniques and those of Information Visualization are integrated (N Space Labs Inc., 2003). 
Relate task: Users need to view relationships between items, as is the case with the LifeLines shown in Figure 3.3, where users can click on a medication and see the related visit report, prescriptions, and laboratory test results. Linking and brushing techniques (Cleveland, 1994) and the Influence Explorer (Tweedie et al., 1996) emphasize the exploration of relationships. Many applications combine multiple visualization techniques that are tightly coupled (for example, (see Roberts, this volume (Chapter 8))). This is a key strength of visualization tools, and a number of chapters in this publication demonstrate examples, particularly those concerned with "Creating Instruments for Ideation" (for example, (see Andrienko et al., this volume (Chapter 5))). Various tools are being developed in various academic and application domains to allow users to specify the visualizations they need and how the interaction between the visualizations should be controlled (North et al., 2002).

History task: It is rare that a single user action produces the desired outcome. Keeping the history of actions allows users to retrace their steps, save useful exploration "recipes” and apply them to updated datasets later on. Roberts, this volume (Chapter 8) considers these issues at an operational level and Gahegan, this volume (Chapter 4) addresses the conceptual, scientific and motivational challenges that underlie support for saving and sharing entire analysis strategies.

Extract and Report task: Users often need to save subsets of the data or particular views of the data into reports. They may also want to publish “cleansed” data with a simplified subset of the tool's features for others to review.

\subsection{The Coming of Age of Information Visualization - Toward Universal Usability}

Although maps have been used for hundreds of years, it is important to realize that most Information Visualization techniques are very recent and, if we exclude elementary tools such as pie or bar charts, these tools have mostly been used by researchers, data analysts and experts. Only recently have we seen novel designs become widely used by the public. Researchers recognize that there is no "one size fits all” visualization and that specialized techniques are needed to tackle special data (for example, genomic data or spatio-temporal data) as well as special user needs (such as collaborative analysis or real time monitoring). Two general challenges need to be faced by Information Visualization before we can anticipate more widespread uptake of many of the techniques that have been developed: the ability to handle larger volumes of data and the ability to effectively support more diverse users. The first challenge relates to the limited number of items most tools available can manage. Many innovative prototypes can only deal with a hundred or a thousand items, or have difficulties maintaining real time interactivity when dealing with larger numbers. Yet examples dealing with millions of items without aggregation have been achieved as shown in Figure 3.6 (Fekete and Plaisant, 2002). Keim et al. (2001) demonstrate that Information Visualization is not yet close to reaching the limits of human visual abilities but that new techniques are needed to tackle the large quantities of information. Keim et al., this volume (Chapter 2) provide an overview and some additional solutions. Dealing with real data also involves data cleansing or dealing with missing and uncertain values. 


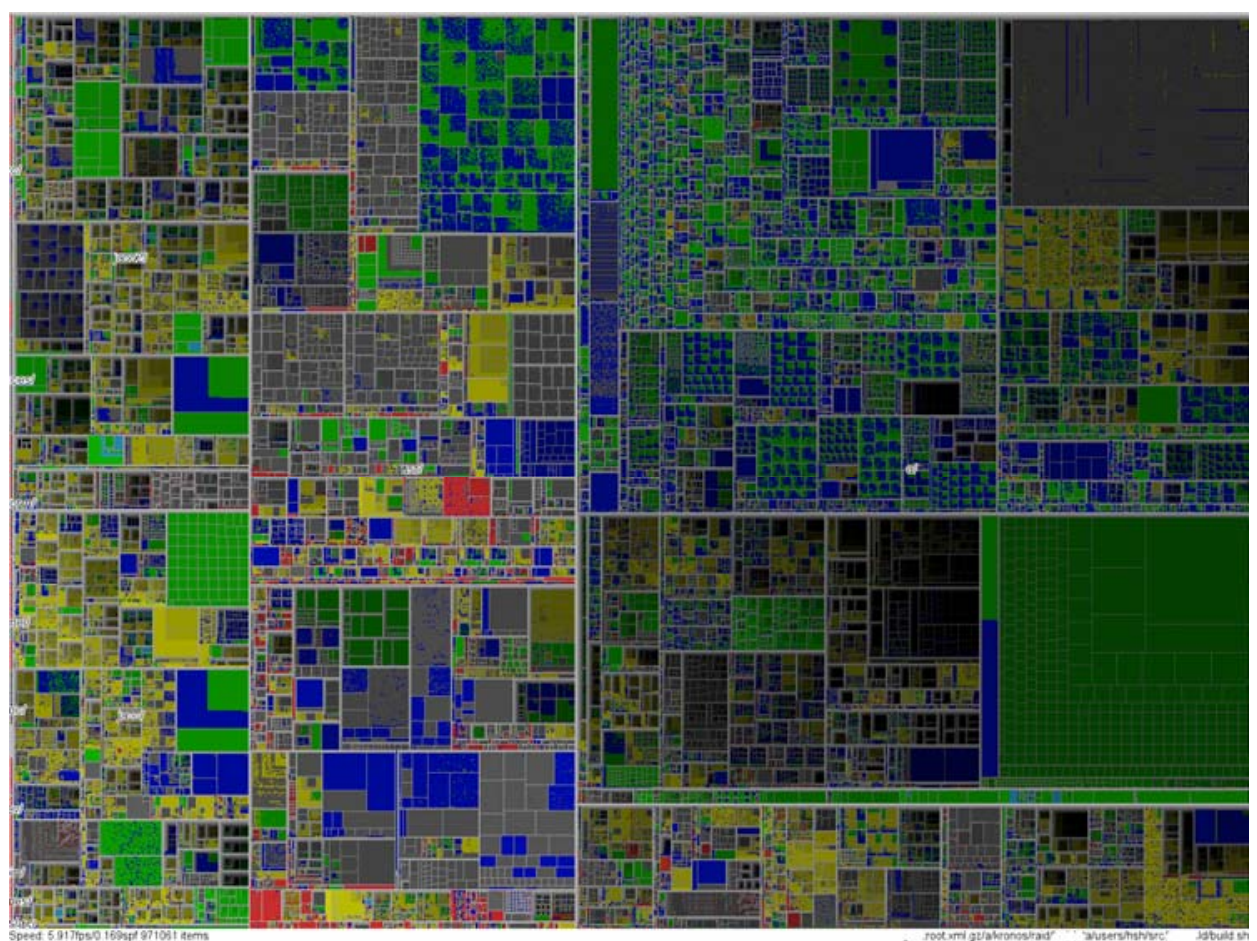

Figure 3.6. A Treemap displaying a million files from a large file system, without aggregation (Fekete and Plaisant, 2002). Careful examination of the high resolution display reveals patterns and special algorithms preserve the interactivity of the rich overviews and filtering tools.

The second challenge is focused on more specifically here: how do we make Information Visualization accessible to a wider group of diverse users? In fact a growing number of applications are being developed for what might be the most challenging user group - the general public. Let us take the example of the enormous amounts of georeferenced data accumulated by government agencies and to be made available to every citizen. These data are useful to senior citizens looking for a place to settle after retirement, business analysts, managers considering relocation, etc. They can also be useful to drivers on the road and researchers working on complex tasks on their high-end computer workstations.

To address the problem of universal usability (Shneiderman, 2000; Shneiderman and Hochheiser, 2001), designers need to consider larger and more diverse groups of users. Technological advances are needed not only to deal with user diversity (age, language, disabilities, etc.) but also with the variety of technology used (screen size, network speed, platform, etc.) and any specific gaps in a user's knowledge (e.g., general knowledge, knowledge of the application domain, of the interface syntax or semantic). Information Visualization has been shown to be a powerful visual thinking or decision tool for some average users receiving a minimum of training (Williamson and Shneiderman, 1992; Lindwarm et al., 1998; Chen and Czerwinski, 2000). But it is becoming important for services to reach and empower every citizen regardless of their background, technical disadvantages or personal disabilities. Universal usability is an ambitious long-term goal but many practical steps can be taken toward achieving it. Focusing on this problem will most likely lead to better technologies for everyone (simpler interfaces, faster downloads, etc). Several examples are reviewed below that illustrate how projects have addressed different aspects of the universal usability challenge.

\subsubsection{Improving general usability}

Of course the first step is to improve the general usability of the interface. Many texts are available to guide designers toward the creation of empowering user interfaces (Shneiderman, 1997; Preece et al., 2002).

Usability testing can then verify that user needs and usability goals have been met and that users are able to use the system on their own with a sense of control and satisfaction (Dumas and Redish, 1999; Kuniavsky, 2003). Specific guidelines for map design are also available (Pickle, 2003) and Tobo'n, this volume 
(Chapter 34) draws upon the usability approach to assess geovisualization map use, for example, (see also Fuhrmann et al., this volume (Chapter 28)). The success of the Map of the Market (Figure 3.4) illustrates that general techniques initially described as "difficult to understand” (i.e., Treemap) can become accessible interfaces. The popularity of this application with the general public might be attributed in part to the fact that the data is familiar, and important, to users who visit a financial website. The careful simplification and refinement of the initial Treemap interface also plays a part. The names and groupings of stocks are familiar to users of the SmartMoney Website. The hierarchy is simple, being shallow and of fixed depth, and the color mapping is natural to most users (using green for gains or increased values of the stocks, red for losses, with alternative colors for those with color impairment). An elegant new algorithm has recently resulted in a better aspect ratio for all the rectangles making the display more readable and pleasing. Labeling and links to details were optimized for the particular application. Finally it was carefully written in lightweight Java so that it could actually run on most users' machines without having to download special plugins or worry about Java versioning. This combination of factors has been effective in making the map usable by the general public, as indicated by the large number of return users the Website enjoys.

\subsubsection{Helping users get started}

Designers need to consider whether their application is to be used by first-time users or by experts who will have invested time and effort into learning the application. A major aspect of accessibility is concerned with enabling users to get started with an application (Andrienko et al., 2002). Even the average professional analyst or researcher may need help, but a major challenge is to help ordinary citizens use Information Visualization applications successfully. Commercial Web sites can decide which population they target but Digital government applications might well be faced by the greatest challenge since they are supposed to be usable by everyone! This implies that most information consumers will be first time users and also that they will have very varied backgrounds and levels of education. They may also have limited time or interest in learning to use the system, but paradoxically may have very high expectations of the services they seek to use. Users want an answer to their question, not necessarily to learn all that a tool can do for them. Let us consider a particular example, that of DataMap (Plaisant, 1993), which was previously named Dynamap or Ymap. Dang et al. (2001) review three approaches that were explored to help users get started with the DataMap interface. DataMap (Figure 3.7) is an interactive visualization tool developed by our Human-Computer Interaction Laboratory at the University of Maryland and is scheduled to be released by the US Census Bureau on CDs and later on the Web. Users can click on a map of the US to display facts about the areas, select multiple areas to compare, zoom on the map or use dynamic queries to filter the map according to a list of criteria. Finally, they can use a scatter plot, tightly coupled to the map and table, to see relationships between two criteria. Our numerous demonstrations and tests in the lab had made us confident that average users could understand and use DataMap after a minute of two of demonstration, but usability tests at the Census Bureau with novice "offthe-street" users receiving no training revealed that many users had difficulties getting started. Some would struggle with certain details of the interface, while others would simply dismiss the interface saying, "It's just too complicated". They had problems not only zooming and selecting multiple regions but also with the sliders. The problems ranged from recognizing that one could move the slider thumbs to understanding what the animation meant. Several users were even puzzled by the interactive scatter plot, some not guessing that dots represented regions and some not knowing how to read a scatter plot at all. The first step was to revise the interface. This was done by a team of colleagues at Virginia Tech led by Chris North and is illustrated in Figure 3.7b. 

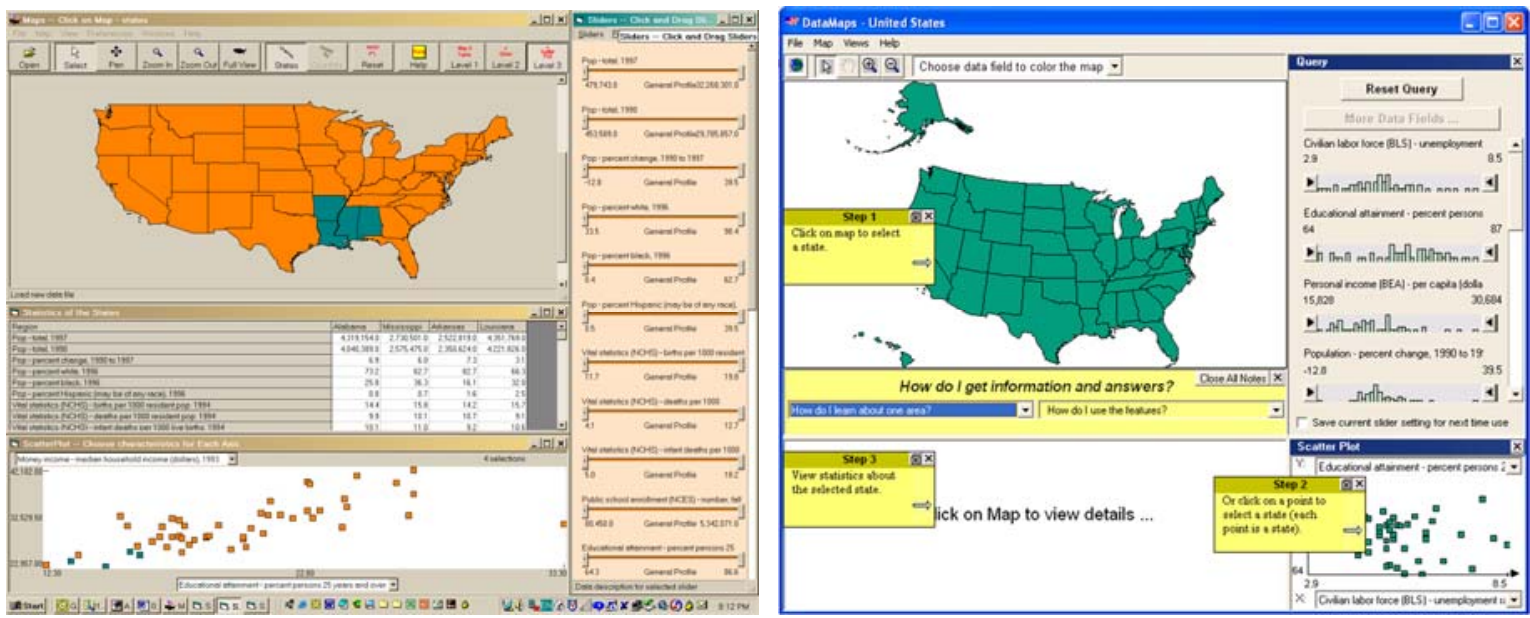

Figure 3.7. (a) DataMap allows users to zoom on a state or county map, select areas or filter them out with dynamic queries. A tightly coupled scatter plot provides an alternative to compare areas. (b) The revised interface (reproduced with permission of Chris North, Virginia Tech).

Most problems found in the usability study led to refinements of the interface. This resulted in a number of changes including: reducing the number of attribute sliders with the possibility of adding more with a control panel; making slider thumbs easier to recognize and use; limiting zooming to avoid zooming into empty areas; simplifying window resizing; using an appropriate projection for the map; using fonts consistently and selecting more readable typefaces; using color coding to show the relationship between the sliders position and the parts of the map highlighted or hidden. Some aspects of the interfaces still needed explanations. Since DataMap is meant for public access, it was clear that users would be unlikely to read manuals or go through long tutorials, so we explored alternative approaches to providing help (Plaisant et al., 2003.) We considered three approaches. The first approach is to create a multi-layered design that provides a simpler interface to get started, while complex features are accessed progressively as users move through the layers of the interface. The second approach is called Integrated Initial Guidance (IIG) or "sticky note help". It provides help within the working interface, right at the start of the application. Using the metaphor of sticky notes overlaid on top of the functional interface it locates the main widgets, demonstrates their manipulation, and explains the resulting actions by replaying recorded sets of actions. The third approach uses video demonstrations of the interface. The videos use sound and entirely overlap the interface to give the illusion of being integrated in the interface ${ }^{1}$.

Approach 1: multi-layered designs Some applications might be suited for a multi-layer design that allows users to start with a simple interface with limited views on information and choices to make and then to move to more complex (and more functional) levels once the basic functions are understood.

This is the case for DataMap, which can easily be organized in a three-layer design (Figure 3.8):

- layer 1: map and table only;

- layer 2: map and table, plus dynamic query filters;

- layer 3: map and table and dynamic query, plus scatter plot.

The interface initial view hides level 2 and level 3 functionality. When users go up one level, the appropriate parts of the interface are added with an animated transition, which help users see what is changing. Multi-layered interfaces, termed "level-structured interfaces" when introduced to Information Visualization (Shneiderman, 1998), are commonly encountered in hardware devices like VCRs that hide advanced function buttons and controls behind a small door, or in search interfaces that often provide a simple and advanced search. This approach has been underused in software applications and could be generalized easily. The benefits are that users can get started more easily. The risk is that some users will never discover the advanced features they do not know are there.

1 Note that this work on Help was done in parallel with Chris North's work on the revision of the interface, so the help techniques are shown on the old interface. Those two projects will be merged eventually. 

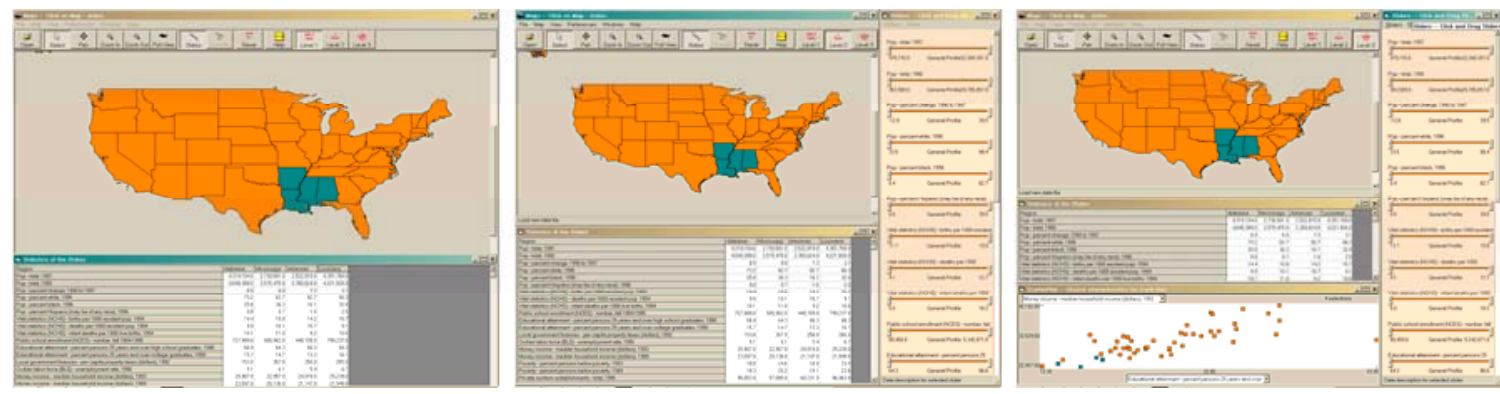

Figure 3.8. Three different levels of the level structured approach. (a) the first level shows only the map and the data table; (b) second level shows slider bar along with map and table; (c) the third level adds scatter plot. An introduction panel lists the features of the level and indicates the location of the three level buttons.

Approach 2: integrated initial guidance or "sticky note help"

IIG implements a metaphor of sticky notes inside the interface itself, thereby allowing users to use the interface or run automated demonstrations while reading the sticky notes overlaid on the functional interface (Figure 3.9). Sticky notes highlight the main functions of the interface, show the location of the main interface widgets whose use can be demonstrated and explained with a "show me" button, and provide lists of simple to complex example tasks which lead to demonstrations of advanced interface widget functions. This allows users to make use of the help in many different ways. Some users will try out all the example tasks, while others may never use any. Some users will use the show me feature while others will execute the steps themselves, guided by the directions on the sticky notes and using the show me function only when they failed to guess what to do.
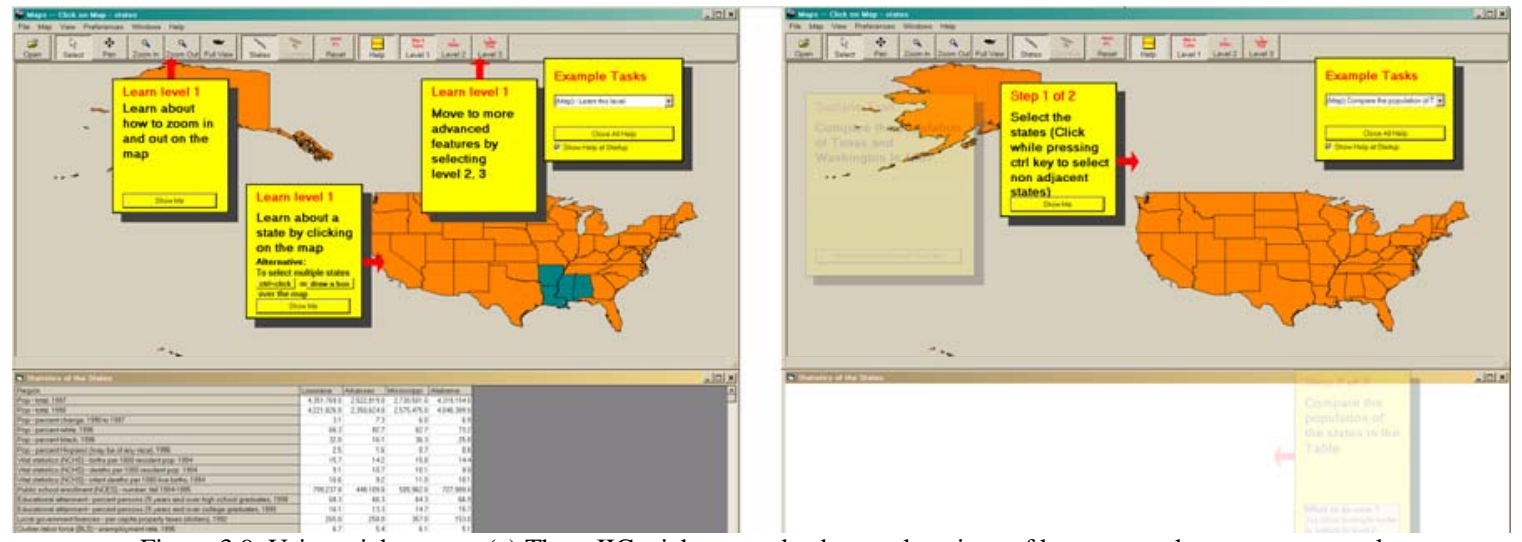

Figure 3.9. Using sticky notes. (a) Three IIG sticky notes lead to explanations of how to use the map, zoom, select multiple states and move to the next level. Example tasks are listed in the note at the top right. (b) The first example task was chosen. "Compare the populations of three west coast states". The next steps are shown in transparency until the user executes the current one, or asks to see an automated "show me" demonstration.

Approach 3: autonomous video or animated demonstrations

Video demonstrations are used increasingly to introduce the main features of complex visual interfaces. They can be launched from the application but are really run outside the interface itself. Macromedia Flash can be used to create demonstrations but simple recording tools (such as Camtasia Studio from TechSmith) are useful to create video explanations in a matter of hours. Effectiveness is increased by the use of speech, which leads to more compact explanations and lively demonstrations (but subtitles will be needed to accommodate users with hearing difficulties). Highlighting the mouse cursor and making mouse clicks or key presses visible is important. Low-resolution videos can be effective and fast to download but we found that videos that entirely cover the interface give users the impression that they are watching the real interface, therefore reducing the transfer of learning to the interface. 
Of these three different approaches, the sticky note technique was found very effective during informal user testing but it was fairly difficult to design and build. On the other hand, the video demonstrations were easier to create and fairly effective when kept short and mapped in direct ways to users tasks. The multilayered approach was appreciated by the majority of our test users and might be the most powerful approach to helping users get started with complex public access applications, as long as care is given to guide users to the advanced levels.

\subsubsection{Addressing the hardware diversity}

Because programmers and development teams usually work with high-end equipmentand fast network connections it is important to address the range of devices and network speed available in people's homes and businesses when producing visualization software for public access. Issues may vary from application to application so we only give an example illustrating how addressing hardware problems does not necessarily result in a "lowest common denominator" applicationbut can benefit everyone.

Let us consider the example of DataMap once again. The Java version of DataMap loaded and ran quickly enough on standard PCs and broadband connections but - as with other interactive map tools - would take minutes to download with a 56K modem and could not maintain the interactivity needed for dynamic queries (i.e., $10 \mathrm{~ms}$ feedback) on low-end PCs. Traditionally, interactive maps use vector graphics to draw the maps which means downloading the vector data and the software to interpret it. Instead, we created a new version of DataMap that encoded geographic knowledge into raster images quickly delivered to the client (Zhao et al., 2003). Algorithms were devised to perform sub-second dynamic query, panning and zooming, with no or minimum server support. This technique leads to download times that were often counted in seconds instead of minutes for modem users, being between 5 and 10 times shorter that those experienced with Web GIS approaches that are based on vector geographic data, while keeping the needed interactivity. The client-side was also implemented as light-weight Java applets to avoid version problems. This example illustrates that it is possible to find technological advances that benefit general public users with slow modem network connections and low-end machines, as well as users with fast T-1 connections and fast machines, therefore advancing the goals of Universal Usability.

\subsubsection{Addressing the needs of users with visual impairments}

\section{Color blindness}

Color impairment is a very common condition that should not be overlooked (Rosenthal and Phillips, 1997; Olson and Brewer, 1997) This problem can more easily be addressed by limiting the use of color, using double encoding when appropriate (for example, by using symbols that vary in both shape and color), providing alternative color palettes to choose from, or allowing users to customize the colors themselves. For example the Map-of-the-Market illustrated in Figure 3.4 provides two choices of color schemes: redgreen and blue-yellow. Various tools are available to both simulate color vision impairments and to optimize graphics for some of the various forms of color impairment that exist, including Vischeck (Dougherty and Wade, 2002). ColorBrewer (Brewer and Harrower, 2002) offers guidelines on color schemes that work for those with color vision impairment.

\section{Low Vision and blindness}

Approximately four million people in the US are blind or visually impaired and it is a requirement for government-provided services to address this population (Vanderheiden, 2000; Muntz et al., 2003). Traditional accommodations to blind and vision-impaired users include the use of speech synthesizers as screen reader, and/or Braille to convey the text information on the display. However, speech-based approaches are very weak at representing 2D spatial layout in the graphical user interface. This is a challenge for maps as well as most other Information Visualization techniques. For navigation in the real world, GPS-based talking maps have been developed (Golledge et al., 1991a,b). One example product is a talking nationwide digital map consisting of most addresses and street intersections (The Sendero Group, 2003). Users can navigate the map using the arrow keys and listen to speech-synthesized descriptions of the map and directions. Trekker (VisuAide, 2003) is another GPS-based application that helps the blind to navigate. Maps are also important to learn an area beforehand and chose 
a route. Schneider and Strothotte (2000) have designed a tangible interface using physical building blocks that users manipulate to promote constructive exploration of the map.

When using maps to learn Geography or discover spatial patterns in data, people with limited vision can use screen magnifiers (for instance, try the "magnifier" available from the Start menu of MS Windows, in the accessibility accessories), but those with severe vision impairments have to rely on tactile maps or atlases (Imperatore, 1992) as shown in Figure 3.10 or sonification of the maps.

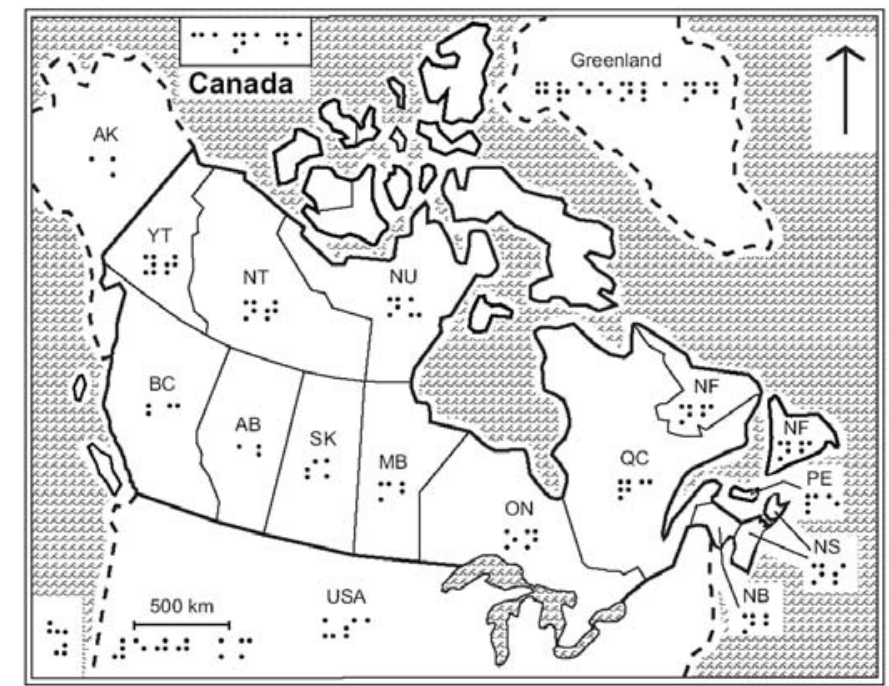

Figure 3.10. A traditional tactile atlas showing a map of Canada. Reproduced with permission of Natural Resources Canada and the Canadian Government.

Current assistive technology research is exploring a number of promising techniques to help blind users benefit from the spatial awareness provided by maps. The first step is to provide data in both visual and descriptive form. OptiMaps (Corda Technologies Inc., 2003), employs a small "d" character below each choropleth map. This is the standard accessibility mark for image "descriptions" that visually impaired users are able to search for. These provide hyperlinks to a textual version of the data, generated automatically with the dynamic map. Examples are available in AtlasPlus (National Cancer Institute, 2003). New vector graphic file formats, such as SVG, permit embedding text descriptions with the graphic information and should simplify this process as a result. Unfortunately reading the data values still does not give an adequate feeling for the spatial relationships between areas that only spatial techniques can provide. Tactile and haptic techniques (and strategies for signifying data haptically) have been devised (Griffin, 2002). Virtual Touch provides an example of map exploration using a tactile mouse. The mouse is equipped with two Braille character mechanical units, which can be used to indicate tactile patterns (using Braille and other alternatives), as shown in Figure 3.11. As users explore the map, the patterns change over different States or areas. One of the limitations of the mouse is that it is an input device providing only relative positioning so absolute position on the map has to be provided by audio feedback.

Another example developed by TouchGraphics and their research colleagues (Landau and Gourgey, 2001) augments standard printed tactile maps. Maps are secured on top of a touch screen that provides the location of each touch (Figure 3.12). The TouchGraphics Atlas has five operational modes. Users can simply explore by touching a physical tactile map and hear names of places touched. They select a destination from an index, and listen to directions that are updated as a user's finger gets closer to the destination. Distance between two points can be calculated, and descriptions of the areas can be listened to. This work was inspired by Nomad (Parkes, 1994). 3D maps can benefit from the use of haptic devices. For example, the Phantom device provides 6 degrees of freedom input and 3 degrees of freedom output to explore the virtual sound space and augment it with haptic feedback providing the same sensation as moving a single finger over a physical 3D map. Tactile maps can be augmented with abstract audio output (Fisher, 1994; Krygier, 1994). For example, users can hear a series of graduated pitches proportional to elevations above sea level as they explore the map. Sonification alone has been demonstrated to be 
effective in giving blind users access to graphs (Ramloll et al., 2001) or, in some limited fashion, entire desktops (Mynatt and Edwards, 1992). In the case of maps, Jeong and Gluck (2002) compared the effectiveness of and preferences for using auditory feedback (volume of sound), haptic feedback (extent of vibration) or both in the tasks of identifying the highest or the middle valued state on a static choropleth map of the US. The experiment showed that overall performance is most successful when using haptic feedback alone but users prefer having both haptic and audio feedback. This result may be attributed to the fact that haptic devices provided spatial cues while the standard sound output does not. Unfortunately tactile and haptic techniques require special hardware, from embossed maps to specialized input devices, and hardware is usually a limiting factor for wide dissemination, as users may not have access to the technology or the financial means to purchase it. Techniques relying solely on sonification have the potential to be used by a larger population as blind users, who often already rely on headphones when using screen readers.

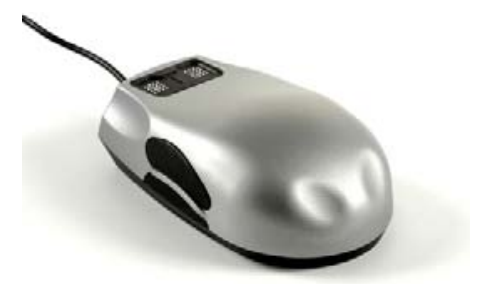

Figure 3.11. The VTPlayer is a mouse equipped with two Braille character units that can also be used to indicate patterns. For example, as users explore a map of the US, patterns change over different states (VirTouch Ltd, 2002). Reproduced with permission of VirTouch Ltd.


Figure 3.12. An example of tactile map augmented with audio description when mounted on a touch screen (Touch Graphics, undated). (a) Sample map plate; (b) the sample map plate in use with the talking tactile tablet (TTT). Reproduced with permission of Touch Graphics.

The BATS project at the University of North Carolina (Parente and Bishop, 2003) uses simple spatial audio (mostly stereo effects) to sonify maps as shown in Figure 3.13. Real world auditory icons are played as the user moves the cursor over the map. For example, car noises are heard over cities and birds and crashing waves are heard over forests and beaches. The sound becomes louder as the user gets close to the source of the sound. Interactivity and immediate feedback provides a pleasant exploration experience and helps blind users explore the map and locate objects. 


\section{North Carolina}



Figure 3.13. The BATS sonic map helps those without sight to explore spatial information (Parente and Bishop, 2003). Real world audio icons (car noises, wave crashing, etc.) are played as the user navigates the map and appear clearly as coming from your right or your left. Volume increases as users get closer to the source of the sound. Reproduced with permission of the authors.

A promising direction for the sonification of spatial information is the use of spatial audio using a headrelated transfer function (HRTF) (Shinn-Cunningham et al., 1997). Since humans are usually able to localize sound with amazing precision by using binaural perception, spatial location can be an important aspect of information perception. It is possible that the addition of positional cues will greatly improve the sonification of maps. Stereo effects allow right-left sound separation only, while spatial audio provides up and down cues, and in a less reliable manner front and back information.

CAVE-like virtual environments provide such spatial sound with sets of multiple loud-speakers (Pape, 1998; see Bodum, this volume (Chapter 19). But synthesized spatial audio allows users to experience 3D audio with ordinary headsets. Until recently this had been limited to high-performance computing environments but researchers are now able to synthesize high-quality 3D sound in real-time on commercial off-the-shelf PCs (Zotkin et al., 2002). We are currently exploring its use for the sonification of maps. Nevertheless, the spatial effects - if noticeable - remain fairly weak and our research at the University of Maryland seeks to combine spatial effects with standard sonification techniques such as those using pitch or tone variations to reinforce the perception of the spatial information. Imagine being in the center of a virtual room with a large US state map hanging from the ceiling and wrapping around you as shown in Figure 3.14. Now imagine that each state on the map "plays" a sound indicating the value of a particular statistics for each state. Because spatial audio is helpful to separate different sounds played together, users can make sense of the location of the states with high, medium and low values. Sonification might also be useful for non-disabled users for monitoring tasks or to represent areas too small to be shown on the map. For example, The District of Columbia is often too small to be visible on US state maps but will be heard in an audio map.

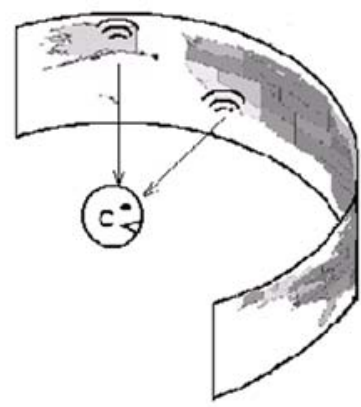

Figure 3.14. Spatial audio allows users to hear the location of virtual sound sources. This schematic represents what a user might hear as each US state "plays" its statistics, for example a sound whose pitch is proportional to its African-American population. Proper linearization of sounds allow users to hear patterns of low to high values moving from the northern states to the lower Mississippi area. 


\subsection{Conclusions}

Information Visualization allows designers to present a large amount of information using abstract representations. Geographic and scientific visualization applications usually use representations determined by the nature of the data being displayed. Location within the graphic is usually used to represent location in space. For example in 2D maps, a projection of space structures the representation, whilst in 3D models of the body or of physical processes such as meteorological predictions graphics are constrained by a 3D locational framework. On the other hand, Information Visualization allows designers to choose among a palette of possible representations that fill space in a variety of ways, such as hierarchies, time lines, networks, tabular displays and the like, to produce information abundant displays. Choosing the appropriate representation(s) is challenging and research is needed to evaluate and compare different approaches. User studies are critical to judge the relative merits of different representations. Tightly coupled displays and highly interactive interfaces using the Zoom, Filter and Detail on Demand principles are needed to allow users to rapidly explore alternative views of the data in a matter of seconds to answer instant queries. Advanced interfaces also need to address the longer term process of analysis that may require annotation, history keeping, collaboration with peers, and the dissemination of results and procedures used. Faster rendering algorithms, sophisticated aggregations techniques to deal with large datasets, and novel labeling techniques are also needed, and along with careful studies of users and their needs will lead to successful visualization applications. Many of these, and related issues are addressed in the following chapters by researchers from a number of related fields.

Information Visualization is becoming increasingly accessible to the general public and attention should be given to the goal of universal usability by enabling the widest range of users to benefit from the applications we develop. Universal usability remains a formidable challenge as we just begin to address the needs of users with slow modems, small screens, or wireless devices. Translation to other languages, access for novice, low education and low motivation users, children and elders all present special difficulties, and users with visual impairments often remain the "forgotten users" of Information Visualization. Tactile solutions are promising for blind users but sonification might provide wider access to map information, as it does not require specialized hardware.

\section{Acknowledgements}

The content of this chapter was inspired in part by Ben Shneiderman's many writings on Information Visualization and Universal Usability, and also by papers co-authored with Haixia Zhao, Hyunmo Kang and other members of the Human-Computer Interaction Laboratory. Some of the work presented was funded by the National Science Foundation under Grant No. EIA 0129978 and the US Census Bureau. I also thank the editors of the book for their careful and extensive editing of the chapter.

\section{References}

Ahlberg, C., Williamson, C., and Shneiderman, B., (1992) "Dynamic queries for information exploration: an implementation and evaluation”, Proceedings ACM CHI’92: Human Factors in Computing Systems. New York: ACM, pp. 619-626.

Andrienko and Andrienko (1999a). Seasonal Migration of White Storks, 1998-1999.Online: http://www.ais.fhg.de/and/java/birds/ (23/10/03).

Andrienko, G., and Andrienko, N., (1999b) "GIS visualization support to the C4.5classification algorithm of KDD”, Proceedings of the 19th International CartographicConference, pp. 747-755.

Andrienko, G., and Andrienko, N., (1999c) “Knowledge-based visualization to supportspatial data mining”, Proceedings of Intelligent Data Analysis. Berlin: SpringerVerlag, pp. 149-160.

Andrienko, G., and Andrienko, N., (1999d) "Making a GIS intelligent: CommonGISproject view”, AGILE’99 Conference, Rome, April 15-17, pp. 19-24.

Andrienko, G. and Andrienko, N., (1999e) Seasonal Migration of White Storks,1998-1999. Online: http://www.ais.fhg.de/and/java/birds/ (03/10/02).

Andrienko, G. L., and Andrienko, N. V., (1999f) “Interactive maps for visual dataexploration”, International Journal Geographic Information Science, 13(4),355-374. 
Andrienko, N., Andrienko, G., Voss, H., Bernardo, F., Hipolito, J., and Kretchmer, U.,(2002) “Testing the usability of interactive maps in CommonGIS”, Cartographicand Geographic Information Science, 29(4), 325-342.

Becker, R. A., and Cleveland, W. S., (1987) “Brushing scatterplots”, Technometrics,29(2), 127-142.

Bederson, B., (1994) "Padpb: advances in multiscale interfaces", Proceedings HumanFactors in Computing Systems CHI ’94 Conference, Boston, MA, p. 315.

Bederson, B., and Shneiderman, B., (2003) The Craft of Information Visualization:Readings and Reflections. San Francisco: Morgan Kaufman.

Bederson, B. B., Meyer, J., and Good, L., (2000) “Jazz: an extensible zoomable userinterface graphics ToolKit in Java, UIST 2000, ACM symposium on user interface software and technology”, CHI Letters, 2(2), 171-180.

Bederson, B. B., Shneiderman, B., and Wattenberg, M., (2002) “Ordered and Quantum treemaps: making effective use of 2D space to display hierarchies”, ACM Transactions on Graphics (TOG), 21(4), 833-854 (Oct. 2002).

Brewer, C. and Harrower, M., (2002) ColorBrewer - Selecting Good Color Schemes for Maps. Online: http://www.personal.psu.edu/faculty/c/a/cab38/ColorBrewerBeta.html (23/10/03).

Card, S. K., Mackinlay, J. D., and Shneiderman, B., (eds.), (1999) Readings in Information Visualization: Using Vision to Think. San Francisco: Morgan Kaufmann Publishers.

Carlis, J., and Konstan, J., (1998) "Interactive visualization of serial periodic data”, Proceedings of User Interface Software and Technology. New York: ACM, pp. 29-38.

Chen, C., (1999) Information Visualisation and Virtual Environments. Berlin: Springer Verlag.

Chen, C., and Czerwinski, M., (2000) "Introduction to the special issue on empirical evaluation of Information Visualizations”, International Journal of Human- Computer Studies, 53(5), 631-635 (Nov. 2000).

Cleveland, W. S., (1994) The Elements of Graphing Data: Revised Edition. Boca Raton, FL: CRC Press.

Cockburn, A., and McKenzie, B., (2002) "Evaluating the effectiveness of spatial memory in 2D and 3D physical and virtual environments", Proceedings ACM CHI 2002 Conference on Human Factors in Computing Systems. New York: ACM Press, pp. 203-210.

Corda Technologies Inc., (2003) Corda Products Optimap 5. Online: http://www.corda.com/products/optimap/ (23/10/03).

Dang, G., North, C., and Shneiderman, B., (2001) "Dynamic queries and brushing on choropleth maps”, Proceedings International Conference on Information Visualization. Piscataway, NJ.: IEEE Press, pp. 757-764.

DiBiase, D., MacEachren, A. M., Krygier, J. B., and Reeves, C., (1992) “Animation and the role of map design in scientific visualization”, Cartography and Geographic Information Systems, 19(4), 201-214, see also pp. 265-266.

Dorling, D., (1992) "Stretching space and splicing time: from cartographic animation to interactive visualization”, Cartography and Geographic Information Systems, 19(4), 215-227, see also pp. 267-270.

Dougherty, R. and Wade, A., (2002) Vischeck. Online: http://www.vischeck.com/ (23/ 10/03).

Dumas, J. S., and Redish, J. C., (1999) A Practical Guide to Usability Testing. Exeter: Intellect Books

Eick, S., Steffen, J., and Sumner, E., (1992) "SeeSoft—a tool for visualizing line-oriented software statistics”, IEEE Transactions on Software Engineering, 18(11), 957-968.

Fekete, J.-D., and Plaisant, C., (1999) "Excentric labeling: dynamic neighborhood labeling for data visualization”, Proceedings of ACM Conference on Human Factors in Computing Systems, CHI '99. New York: ACM Press, pp. 512-519.

Fekete, J., and Plaisant, C., (2002) “Interactive Information Visualization of a million items”, Proceedings of IEEE Conference on Information Visualization, Boston, Sept. 2002, pp. 117-124.

Fisher, P., (1994) "Hearing the reliability in classified remotely sensed images", Cartography and Geographic Information Systems, 21(1), 31-36.

Friendly, M., (1994) "Mosaic displays for multi-way contingency tables”, Journal of the American Statistical Association, 89(425), 190-200.

Furnas, G. W., (1986) “Generalized fisheye views”, Proceedings CHI’86 Conference: Human Factors in Computing Systems. New York: ACM Press, pp. 16-23.

Gahegan, M., Takatsuka, M., Wheeler, M., and Hardisty, F., (2002a) “GeoVISTA Studio: a geocomputational workbench, Computers”, Environment and Urban Systems, 26, 267-292. 
Gahegan, M., Takatsuka, M., Wheeler, M., and Hardisty, F., (2002b) “Introducing GeoVISTA Studio: an integrated suite of visualization and computational methods for exploration and knowledge construction in geography”, Computers, Environment and Urban Systems, 26(4), 267-292.

Golledge, R. G., (1991) “Tactual strip maps as navigational aids”, Journal of Blindness and Vision Impairment, 85(7), 296-301.

Golledge, R. G., Loomis, J. M., Klatzky, R. L., Flury, A., and Yang, X. L., (1991) “Designing a personal guidance system to aid navigation without sight: progress on the GIS component”, International Journal of Geographical Information Systems, 5(4), 373-395.

Griffin, A., (2002) "Feeling it out: the use of haptic visualization for exploratory geographic analysis", Cartographic Perspectives, 39, 12-29.

Grosjean, J., Plaisant, C. and Bederson, B., (2002) SpaceTree. Online: http://www.cs.umd.edu/hcil/spacetree (23/10/03).

Imperatore, W., (1992) The Braille World Atlas. Louisville, Kentucky: American Printing House for the Blind, Inc.

Inselberg, A., (1985) “The plane with parallel coordinates”, The Visual Computer, 1, 69-91.

Inselberg, A., (1997) “Multidimensional detective”, Proceedings of IEEE Symposium on Information Visualization (InfoVis '97), Phoenix, AZ, Oct. 1997, pp. 100-107.

Inxight Software Inc., (2002) Inxight Software - Unstructured Data Management Solutions. Online: http://www.inxight.com (23/10/03).

Jeong, W., and Gluck, M., (2002) "Multimodal bivariate thematic maps with auditory and haptic display”, Proceedings of 2002 International Conference on Auditory Display, Kyoto, Japan, pp. 1-4.

Johnson, B., and Shneiderman, B., (1991) "Tree-maps: a space-filling approach to the visualization of hierarchical information structures”, Proceedings IEEE Visualization '91. Piscataway, NJ: IEEE, pp. 284-291.

Keim, D. A., (2001) "Visual exploration of large data sets”, Communications of the ACM, 44(8), 38-44.

Keim, D. A., and Kriegel, H.-P., (1994) “Database exploration using multidimensional visualization”, Computer Graphics \& Applications, 6, 40-49, (September 1994).

Kraak, M. J., (undated) Minard - What has Been Done by ITC? Online: http://www.itc.nl/personal/kraak/1812/minard-itc.htm (23/10/03).

Kraak, M. J., (1989) “Computer-assisted cartographic three-dimensional imaging techniques”, In: Raper, J., (ed.), Three Dimensional Applications in Geographical Information Systems. London: Taylor \& Francis, pp. 99-114.

Kraak, M. J., and MacEachren, A. M., (1994) "Visualization of the temporal component of spatial data”, In: Waugh, T., and Healey, R., (eds.), Proceedings of the Sixth International Symposium on Spatial Data Handling, Advances in GIS Research, Edinburgh, Scotland, pp. 391-409.

Krygier, J., (1994) “Sound and geographic visualization”, In: MacEachren, A. M., and Taylor, D. R. F., (eds.), Visualization in Modern Cartography. Oxford, UK: Pergamon, pp. 146-166.

Kuniavsky, M., (2003) Observing the User Experience: A Practitioner’s Guide to User Research. San Francisco: Morgan Kaufmann.

Kwan, M. P., (2000) "Interactive geovisualization of activity-travel patterns using 3D geographical information systems: a methodological exploration with a large data set”, Transportation Research Part C-Emerging Technologies, 8(1-6), 185-203.

Lamping, J., Rao, R., and Pirolli, P., (1995) “A focus p context technique based on hyperbolic geometry for visualizing large hierarchies”, Proceedings of CHI '95 - Human Factors in Computing Systems, Denver, CO, May 1995. New York: ACM Press, pp. 401-408.

Landau, S., and Gourgey, K., (2001) “Development of a talking tactile tablet”, Information Technology and Disabilities, VII(2).

Lindwarm, D., Rose, A., Plaisant, C., and Norman, K., (1998) “Viewing personal history records: a comparison of tabular format and graphical presentation using LifeLines”, Behaviour \& Information Technology, 17(6), 249-262.

MacEachren, A. M., Dai, X., Hardisty, F., Guo, D., and Lengerich, G., (2003a) "Exploring high-D spaces with multiform matrices and small multiples”, IEEE Symp. on Information Visualization, pp. 31-38.

MacEachren, A. M., Hardisty, F., Dai, X., and Pickle, L., (2003b) “Geospatial statistics supporting visual analysis of federal GeoSpatial statistics”, Digital Government Table of Contents, pp. 59-60.

Muntz, R. R., Barclay, T., Dozier, J., Faloutsos, C., MacEachren, A. M., Martin, J. L., Pancake, C. M., and Satyanarayanan, M., (2003) "IT roadmap to a geospatial future”, Report of the Committee on 
Intersections Between Geospatial Information and Information Technology. Washington, DC: National Academy of Sciences.

Mynatt, E., and Edwards, K., (1992) "Mapping GUIs to auditory interfaces”, Proceedings of the 5th Annual ACM Symposium on User Interface Software and Technology. New York: ACM Press, pp. 61-70.

N Space Labs Inc., (2003) incMape: Geographic Map of the Market. Online: http://www.nspacelabs.com/ (23/10/03).

National Cancer Institute, (2003) Cancer Mortality Maps and Graphs. Online: http://www3.cancer.gov/atlasplus/ (23/10/03).

Nielson, G. M., Hagen, H., and Mueller, H., (1997) Scientific Visualization: Overviews, Methodologies, Techniques. Piscataway, NJ: IEEE Computer Society.

North, C., Conklin, N., and Saini, V., (2002) "Visualization schemas for flexible Information Visualization", Proceedings of InfoVis 2002, pp. 15-22.

Olson, J., and Brewer, C. A., (1997) "An evaluation of color selections to accommodate maps users with color-vision impairments”, Annals of the Association of American Geographers, 87(1), 103-134.

OmniViz Inc., (2003) Omniviz. Online: http://www.omniviz.com (23/10/03).

Pape, D., (1998), “Crayoland”. In: SIGGRAPH Video Review, Issue 127/SIGGRAPH '98 Electronic Art and Animation Catalog CD-ROM. Orlando, FL: ACM. Online: http://www.evl.uic.edu/pape/projects/crayoland.

Parente, P., and Bishop, G., (2003) "BATS: the blind audio tactile mapping system”, Proceedings of ACM South Eastern Conference, Savannah, GA, http://www.cs.unc.edu/Research/assist/bats/history.shtml (Mar. 2003).

Parkes, D., (1994) "Audio tactile systems for designing and learning complex environments as a vision impaired person: static and dynamic spatial information access”, In: Steele, J., and Hedberg, J. G., (eds.), Learning Environment Technology: Selected Papers from LETA 94. Canberra, AJET Publications, pp. 219-223.

Perlin, K., and Fox, D., (1993) "Pad: an alternative approach to the computer interface”, In: Kajiya, J. T., (ed.), Proceedings of the 20th Annual ACM Conference on Computer Graphics (SIGGRAPH '93), Aug. 2-6, Anaheim, CA. New York: ACM Press, pp. 57-64.

Pickle, L., (2003) "Usability testing of map designs”, Proceedings of Interface 2003. Alexandria, VA: ASA.

Plaisant, C., (1993) "Facilitating data exploration: dynamic queries on a health statistics map", Proceedings of the Annual Meeting of the American Statistical Association of the Government Statistics Section, San Francisco, CA, Aug 18-23. Alexandria, VA: ASA pp. 18-23.

Plaisant, C., Rose, A., Milash, B., Widoff, S., and Shneiderman, B., (1996) "LifeLines: visualizing personal histories”, Proceedings of CHI '96 Conference: Human Factors in Computing Systems. New York: ACM, pp. 221-227, 518.

Plaisant, C., Grosjean, J., and Bederson, B., (2002) "SpaceTree: supporting exploration in large node link tree, design evolution and empirical evaluation”, Proceedings of IEEE Conference on Information Visualization, pp. 57-64.

Plaisant, C., Kang, H., and Shneiderman, B., (2003) "Helping users get started with visual interfaces: multilayered interfaces, integrated initial guidance and video demonstrations”, Proceedings of HumanComputer Interaction International 2003, Crete, June 2003, pp. 790-794.

Preece, J., Rogers, Y., and Sharp, H., (2002) Interaction Design: Beyond Human- Computer Interaction. New York: Wiley.

Ramloll, R., Brewster, S., Yu, Y., and Riedel, B., (2001) "Using non-speech sounds to improve access to 2D tabular numerical information for visually impaired users”, Proceedings 15th British HCI Group Annual Conference on Human-Computer Interaction (IHMHCI), Lille, France, Sept. 10-14.

Rao, R., and Card, S. K., (1994) "The table lens: merging graphical and symbolic representation in an interactive focus b context visualization for tabular information”, Proceedings Human Factors in Computing Systems CHI '94 Conference, Boston, MA. New York: ACM, pp. 318-322.

Rosenthal, O., and Phillips, R., (1997) Coping With Color-Blindness. New York: Avery Publishing Group. Schneider, J., and Strothotte, T., (2000) "Constructive exploration of spatial information by blind users", Proceedings of the ACM Conference on Assistive Technologies, ASSETS 2000. New York: ACM, pp. 188-192.

Shinn-Cunningham, B. G., Lehnert, H., Kramer, G., Wenzel, E. M., and Durlach, N. I., (1997) “Auditory displays”, In: Gilkey, R., and Anderson, T., (eds.), Binaural and Spatial Hearing in Real and Virtual Environments. New York: Erlbaum, pp. 611-663. 
Shneiderman, B., (1997) Designing the User Interface - Strategies for Effective Human- Computer Interaction. Reading: Addison-Wesley Publishing Company, p. 639.

Shneiderman, (1998) Treemaps for Space-Constrained Visualization of Hierarchies. Online: http://www.cs.umd.edu/hcil/treemaps (23/10/03).

Shneiderman, B., (2000) "Universal usability: pushing human-computer interaction research to empower every citizen”, CACM, 23(5), 84-91.

Shneiderman, B., (2001) "Supporting creativity with advanced information-abundant user interfaces”, In: Earnshaw, R., Guedj, R., Van Dam, A., and Vince, J., (eds.), Human-Centred Computing, Online Communities, and Virtual Environments. London: Springer-Verlag, pp. 469-480.

Shneiderman, B., and Hochheiser, H., (2001) "Universal usability as a stimulus to advanced interface design”, Behaviour\&Information Technology, 20(5), 367-376, Online: http://www.otal.umd.edbu/uupracticechen

SmartMoney.com, (2003) Smartmoney.com - Investing, Saving and Personal Finance. Online: http://www.smartmoney.com (23/10/03).

Spence, R., (2001) Information Visualization. Harlow: Addison Wesley/ACM Press Books, 206 pp.

Szego, J., (1987) Human Cartography:Mapping theWorld ofMan. Stockholm:Almqvist\& Wiksell International, p. 236.

The Hive Group Inc., (2003) The Hive Group - Creators of Honeycomb Technology. Online: http://www.hivegroup.com (23/10/03).

The Sendero Group, (2003) Sendero Group Homepage - GPS for the blind. Online: http://www.senderogroup.com

Theus, M., (2002a) “Geographical Information Systems”, In: Kloesgen, W., and Zytkow, J., (eds.), Handbook of Data Mining and Knowledge Discovery. Oxford: Oxford University Press.

Theus, M., (2002b) “Interactive data visualization using Mondrian”, Journal of Statistical Software, 7(11).

Tufte, E. R., (1983) The Visual Display of Quantitative Information. Cheshire, CT: Graphics Press, p. 197.

Tweedie, L., Spence, R., Dawkes, H., and Su, H., (1996) “Externalising abstract mathematical models”, In: Bilger, R., Guest, S., and Tauber, M. J., (eds.), CHI '96: Conference on Human Factors in Computing Systems, Vancouver, BC, ACM/ SIGCHI, Online: http://www.acm.org/sigs/sigchi/chi96/proceedings/papers/Tweedie/lt1txt.htm

Vanderheiden, G., (2000) "Fundamental principles and priority setting for universal usability", Proceedings ACM Conference on Universal Usability 2000. New York: ACM, pp. 32-38.

VirTouch Ltd., (2002) Innovative Tactile Solutions for PC Users Who Are Blind. Online: http://www.virtouch.com/ (23/10/03).

VisuAide, (2003) GPS Orientation Solutions - Trekker. Online: http://www.visuaide.com/gpssol.html $(23 / 10 / 03)$

Ware, C., (2000) Information Visualization: Perception for Design. San Francisco: Morgan Kaufmann Publishers, p. 384.

Weibel, R., and Jones, C. B., (1998) “Computational perspectives on map generalization”, Geoinformatica, 2(4), 307-314.

Williamson, C., and Shneiderman, B., (1992) "The dynamic home finder: evaluating dynamic queries in a real-estate information exploration system”, Proceedings of the ACM SIGIR'92 Conference. New York: ACM, pp. 338-346.

Wise, T. A., Thomas, J. J., Pennock, K., Lantrip, D., Pottier, M., Schur, A., and Crow, V., (1995) "Visualizing the non-visual: spatial analysis and interaction with information from text documents", Proceedings IEEE Information Visualization '95, Los Alamitos, CA. Piscataway, NJ: IEEE Computer Press, pp. 51-58.

Woodruff, A., Landay, J., and Stonebreaker, M., (1998) "Constant information density in zoomable interfaces”, Proceedings of the 4th International Working Conference on Advanced Visual Interfaces AVI '98, L'Aquila, Italy, May 24-27, pp. 110-119.

Zhao, H., Plaisant, C., and Shneiderman, B., (2003) "Improving accessibility and usability of georeferenced statistical data”, Proceedings 2003 National Conference on Digital Government Research, Online: http://www.dgrc.org/dgo2003

Zotkin, D. N., Duraiswami, R., and Davis, L. S., (2002) “Creation of virtual auditory spaces”, Proceedings International Conference on Acoustic Speech and Signal Processing, Orlando, FL, May 2002, pp. 21132116. 